\title{
FETICHISMO VIRTUAL NA VIDA DE UM HIKIKOMORI BRASILEIRO: UM ESTUDO DE CASO
}

\author{
FETICHES VIRTUALES EN LA VIDA DE UN HIKIKOMORI BRASILEÑO: UN \\ ESTUDIO DE CASO
}

\author{
VIRTUAL FETISH IN THE LIFE OF A BRAZILIAN HIKIKOMORI: A CASE \\ REPORT
}

\author{
Cláudia Dias PRIOSTE ${ }^{1}$ \\ Ranyella Cristina de SIQUEIRA ${ }^{2}$
}

RESUMO: O fenômeno hikikomori, identificado no Japão nos anos de 1990, é uma condição psicossocial em que a pessoa, deliberadamente, se isola da sociedade por seis meses ou mais. Trata-se de um problema crescente no mundo. $\mathrm{O}$ caso descrito nesse estudo refere-se a um homem de 38 anos que havia abandonado o emprego há mais de dez anos e se mantido em isolamento social por mais de cinco. Sua rotina consistia em acessar jogos e páginas pornográficas na internet. A análise deste caso destacou alguns fatores psicodinâmicos que podem estar relacionados a um maior risco para o isolamento social: base narcísica frágil, fixação edipiana materna e dificuldade em lidar com conflitos na idade escolar. Além disso, destacou-se a hiperestimulação de fantasias virtuais, com ênfase no fetichismo, voyeurismo e sadomasoquismo.

PALAVRAS-CHAVE: Hikikomori. Isolamento social. Fetichismo. Fantasia virtual. Cibercultura. Psicanálise.

RESUMEN: El fenómeno hikikomori, identificado en Japón en la década de 1990, es una condición psicosocial en que la persona permanece completamente aislado de la sociedad por seis meses o más. Este es un problema creciente en el mundo. El caso descrito en este estudio es sobre un hombre de 38 años que había dejado su trabajo por más de diez años y permaneció en aislamiento social por más de cinco años. Su rutina consistía en acceder a juegos virtuales y páginas pornográficas en Internet. El análisis de este caso destacó algunos factores psicodinámicos que pueden estar relacionados con mayor riesgo de aislamiento social: una base narcisista frágil, la fijación edípica materna y la dificultad para lidiar con los conflictos escolares. Además, se destacó la hiperestimulación de fantasías virtuales, con énfasis en el fetichismo, el voyerismo y el sadomasoquismo.

PALABRAS CLAVE: Hikikomori. Aislamiento social. Fetichismo. Fantasía virtual. Cibercultura. Psicoanálisis.

${ }^{1}$ Universidade Estadual Paulista (UNESP), Araraquara - SP - Brasil. Docente do Departamento de Psicologia da Educação. ORCID: https://orcid.org/0000-0001-6824-3410. E-mail: claudiaprioste@ gmail.com

${ }^{2}$ Universidade Estadual Paulista (UNESP), Araraquara - SP - Brasil. Mestranda do Programa de Pós-Graduação em Educação Sexual. ORCID: https://orcid.org/0000-0003-3432-5498. E-mail: ranysiqueira@ gmail.com

Doxa: Rev. Bras. Psico. e Educ., Araraquara, v. 21, n. 1, p. 4-16, jan./jun., 2019. e-ISSN: 2594-8385. 
ABSTRACT: The hikikomori phenomenon, identified in Japan in the 1990s, is a psychosocial condition in which a person completely withdraws from society for six months or more. This problem has been increasing in several countries. The case reported in this paper is about a 38-year-old man that had withdrawn from work from more than ten years and kept isolated in his bedroom for more than five years. He used to play online games and access pornography websites in his daily routine. This case evidences the combination of factors that may have contributed to increasing the risk of social isolation: fragile narcissistic foundations, maternal oedipal fixation, difficulties to manage conflicts at scholar phase. Besides, it highlights the excessive fetishist, voyeuristic and sadomasochistic excitement with the internet.

KEYWORDS: Hikikomori. Social withdrawal. Fetishism. Virtual fantasies. Cyberculture. Psychoanalysis.

\section{Introdução}

O surgimento da expressão hikikomori para descrever um fenômeno identificado nos jovens japoneses no final dos anos de 1990 (SAITO, 1998) não parece ser uma coincidência quando relacionado à globalização e à popularização da internet, que ocorreu no mesmo período. Não somente no Japão, como em diversos país, tem sido identificado um aumento de jovens enclausurados em seus quartos por meses ou anos, com pouco contato externo, sem frequentar escola ou trabalho, vivendo apenas do suporte financeiro de seus pais (KATO et al., 2012; BOMMERSBACH; MILLARD, 2019; BOWKER et al., 2019).

Hikikomori refere-se a um fenômeno social e psicológico em que a pessoa se encontra em isolamento social por mais de seis meses (SAITO, 1998; KANEKO, 2006). Kato, Kanba e Teo (2019) propõem a seguinte redefinição do conceito de hikikomori: trata-se de um isolamento social patológico, por no mínimo seis meses, sobretudo de forma física, com significativo comprometimento funcional ou sofrimento em decorrência do isolamento. De acordo com Suwa e Suziki (2012), esse problema é frequentemente identificado entre os homens.

Nos últimos anos, diversos artigos têm sido produzidos com objetivo de descrever o fenômeno, suas causas, além de discutir até que ponto podemos considerá-lo uma nova psicopatologia (LI; WONG, 2015; KATO; KANBA; TEO, 2019). Por outro lado, há divergências no que se refere à dimensão conceitual e etiológica. Nos estudos sociológicos são prevalentes as explicações a respeito da oposição entre os valores conformistas da sociedade japonesa e o contexto da globalização (TOIVONEN et al, 2011). Alguns estudos chamam a atenção para a recorrente superproteção dos pais nos 
casos identificados de hikikomori (TEO, 2010). Outras pesquisas relacionam síndrome hikikomori às altas exigências acadêmicas (SUWA; SUZIKI, 2013) como também aos processos de rejeição, disputa e bullying no ambiente escolar (TEO, 2010). Entre os psiquiatras, prevalecem as explicações sobre os distúrbios de humor e personalidade (KATO et al., 2012).

A despeito do interesse sobre esta problemática ter aumentado, ainda são poucos os estudos que envolvem uma compreensão mais ampla deste fenômeno. Além disso, as dificuldades metodológicas merecem destaque, em especial por se tratar de pessoas que não gostam de revelar seus sentimentos (LI; WONG, 2015). Quando se trata de Brasil, em pesquisas realizadas nas bases de dado Scielo, Capes e Pub Med com as palavras-chaves 'hikikomori' ou 'severe social isolation', identificamos somente dois artigos a respeito do tema, um no idioma inglês e outro em portugues. A pouca produção brasileira de pesquisas sobre o tema, provavelmente, está relacionada aos diferentes diagnósticos psiquiátricos, além de ser um fenômeno silencioso e recente.

No artigo "Hikikomori in Brazil: 29 anos de voluntário isolamento social" Gondim, Aragão, Holanda Filha, Messias (2017) descreve o caso de isolamento social de um homem durante 29 anos e, sua recuperação espontânea tardia. Os autores sustentam que se trata de um caso de hikikomori em função da similaridade com as características clássicas identificadas no Japão: homem jovem de classe média, diante de intensos estressores sociais e inadequado suporte psiquiátrico e familiar. O artigo 'Hikikomori: revisão sobre um grave fenômeno de isolamento social' foi o primeiro trabalho sobre este tema em língua portuguesa no Brasil. Domingues-Castro e Torres (2018) apresentam uma revisão de literatura sobre o fenômeno hikikomori e destacam o aumento dos casos como uma preocupação internacional. Afirmam que este fenômeno está presente em diversos países europeus, asiáticos e americanos, com características clínicas similares às relatadas no Japão.

Nos artigos sobre hikikomori, revisados nesta pesquisa, foram constatadas lacunas no que concerne às descrições dos hábitos virtuais e preferências sexuais das pessoas com esse diagnóstico. Vale destacar que este fenômeno foi inicialmente relatado no Japão, onde tem sido próspera a indústria pornográfica e seus fetichismos, como mencionado por Dufour (2011). A despeito disto, pouca atenção tem sido dedicada à sexualidade e aos hábitos virtuais relacionados dos hikikomoris.

No início dos anos 2000, Prioste (2016) observou um aumento no número de casos de adolescentes homens com tendência ao isolamento social e problemas associados ao uso intensivo da navegação na internet para fins de jogos e pornografia. Nós também supomos 
similar tendência entre os adultos, e um desses casos se destacou devido ao severo isolamento social apresentado.

Nesse contexto, este artigo tem o objetivo de analisar a influência da internet nas satisfações sexuais substitutivas, em especial, do fetichismo sexual e sua relação ao processo de isolamento social de um caso, semelhante ao descrito na literatura científica como hikikomori. O caso aqui apresentado será denominado Wilson ${ }^{3}$. Esta análise também teve o objetivo de propor novas hipóteses a serem investigadas, considerando possíveis fatores de risco para o fenômeno a fim de contribuir para a prevenção do isolamento social entre os jovens.

\section{Fundamentação teórica}

Freud (1905) chocou a comunidade científica quando propôs a existência de uma sexualidade perverso-polimorfa na infância, questionando as fronteiras entre $o$ 'perverso' e o 'normal' no âmbito da constituição psíquica. Diferente da sexualidade adulta, a qual tem a relação sexual como foco, a sexualidade infantil pode ser considerada polimorfa, no sentido de satisfações substitutivas sem finalidade de intercurso genital, com destaque para as satisfações voyeurístico-exibicionistas e sadomasoquistas, presente em idade precoce. Essa flexibilidade pulsional seria uma vantagem do ser humano quando comparado a outros animais, pois na ausência de um objeto fixo de satisfação, seria possível um deslocamento da pulsão sexual para criações culturalmente significativas.

Se no tempo de Freud, a moral civilizada incitou a repressão das perversões polimorfas e as direcionou à manutenção da força produtiva por meio da sublimação (FREUD, 1930), na sociedade de consumo, as fantasias perversas passaram a ser economicamente exploradas (DUFOUR, 2009). Nesse contexto, é preciso questionar a predominância do que Lebrun (2007) denomina perversões ordinárias ${ }^{4}$ dos neosujeitos. A diferença entre a perversão clássica e a perversão ordinária seria relacionada ao processo de subjetivação: enquanto o neo-sujeito seria resistente à subjetivação e permaneceria atrelado às fantasias perverso-polimorfas, o perverso assume a negação

${ }^{3} \mathrm{O}$ nome do paciente e o objeto de seu fetiche foram mudados para assegurar seu anonimato. Esse nome foi escolhido em decorrência da identificação do paciente com o filme "O Náufrago" (2001), nas cenas em que o protagonista conversava com uma bola que ele chamava de Wilson.

44 Perversão comum: essa seria uma curta perversão produzida no dia-a-dia e estimulada pelo contexto socialeconômico: "uma perversão econômica pode envolver os indivíduos em um vínculo ou negação de maneira que os indivíduos não são propriamente perversos" (LEBRUN, 2007, p. 333, tradução nossa). 
(Verleugnung) como um modo subjetivação (LEBRUN, 2007). Importante salientar que, os neo-sujeitos tendem a permanecer excessivamente vinculados a mãe, se recusando a amadurecer e ter responsabilidades da vida adulta, tal como ocorreu com Wilson e talvez ocorra com outros casos identificados como hikikomori.

\section{Uma cultura fetichista}

Freud (1905) definiu fetichismo como um mecanismo de satisfação obtido por meio da substituição do objeto sexual por uma parte do corpo ou um objeto inanimado. Em seu ponto de vista, o caso pode ser considerado patológico se o fetiche se torna a única forma de satisfação sexual. Birman (2009) observou que Freud (1927) redefiniu o conceito de perversão ao esclarecer a constituição do objeto fetiche no psiquismo masculino em relação à ao mecanismo de negação (Verleugnung). Portanto, "a perversão se destaca, nesse contexto teórico, como sendo algo fundamentalmente masculino, como resultado da angústia de castração, que seria colocada em discussão nesse tipo de experiência sexual" (BIRMAN, 2009, p. 383). Embora Freud (1927) tenha iniciado seu trabalho a partir de uma teoria infantil, o fetiche é mais do que uma tentativa de imaginar um falo onde não existe nenhum, trata-se de um modo específico de lidar com experiências de privação.

Türcke (2011), ao retomar à análise de Freud, estabelece a relação entre um suposto trauma original e a necessidade de repetição, como uma tentativa de lidar com a angústia. $\mathrm{Na}$ visão do autor, a cultura contemporânea tem criado padrões de excitações para lidar com a angústia existencial, a qual estabelece um sintoma abstinência generalizada e, concomitantemente, um paradigma pautado na busca por sensações, com fixação em objetos fetichistas.

Segundo Dufour (2009), o projeto de uma sociedade perversa está sendo criado há séculos, e Sade foi um dos grandes precursores, com Mandeville e Adam Smith, ao afirmarem a supremacia lógica do vício privado como benéfico para a economia. No entanto, no início do século XX, um passo decisivo foi tomado no sentido de que o fetichismo seria um tipo de exploração econômica indireta no que tange à sexualidade. Edward Barnays, sobrinho de Freud, destacou-se por usar o conhecimento psicanalítico em uma campanha publicitária em Nova York durante a crise de 1929. Neste evento, mulheres atraentes desfilaram fumando cigarros, em alusão à felação, com a mensagem: 'tochas da liberdade'. A proposta da propaganda era de substituição inconsciente da satisfação sexual pelo cigarro. Isto também incluía uma alusão à conquista da liberdade feminina (DUFOUR, 2009). 
Se no período da industrialização, bebidas alcóolicas eram distribuídas nas fábricas como meio de alívio e distração aos trabalhadores devido ao ônus dos intensivos turnos de trabalho (TÜRCKE, 2011), no século passado, o cinema e o entretenimento televisivo também passaram a fazer parte das satisfações sexuais substitutivas (ADORNO; HOKEIMER, 2002). Atualmente, o ciberespaço ao reunir mídias, mercado, redes sociais, jogos e pornografias, se tornou um ambiente propício para excitamento e exploração de fantasias perverso-polimorfas e fetichismos (PRIOSTE, 2016).

\section{Descrição do caso}

Wilson tinha 38 anos quando foi encaminhado para atendimento psicológico, após ter sido atendido em serviço de urgência devido a uma dor torácica intensa. Em sua primeira consulta demonstrou apatia, pensamento lento, dificuldade de organização temporal e preocupantes lapsos de memória. Não sabia dizer há quanto tempo estava sem sair de casa, talvez cinco anos ou mais. Também não conseguia se lembrar da última vez em que esteve no trabalho. Sua rotina, por um longo tempo, consistia em trocar dias por noites, navegar na internet, assistir animes, tomar café e jogar no computador. Ele morava em uma casa humilde e seu pai aposentado o sustentava financeiramente. Wilson, no final da primeira sessão, revelou uma falta de sentido em sua vida: 'viver ou morrer ... tanto faz'.

O paciente negou ter pensamentos suicidas e disse que, antes desta crise, ele estava satisfeito com o seu modo de viver, se considerava uma pessoa sem ambição. Contou que gostava de navegar na internet, pois assim, não se sentia melancólico ou entediado. Em suas palavras: "se eu tivesse dinheiro para comprar cigarros, eu não estaria aqui em busca de tratamento. Estava tudo bem". Familiares e alguns amigos estavam preocupados com seu isolamento, embora ele afirmasse que se sentia melhor sem contato com pessoas, inclusive no ambiente virtual.

Seu processo de isolamento foi progressivo, primeiro abandonou o trabalho, depois abandonou os contatos pessoais com os amigos, mantendo somente conversas on-line. Nos últimos anos, deixou de responder e-mail ou qualquer mensagem enviada pelo restrito círculo de amigos que tinha. Seu contato com os familiares na mesma casa também foi se tornando mais limitado. 
Wilson começou a trabalhar quando era adolescente e, aos 20 anos, conseguiu uma vaga na área de Tecnologia da Informação (T.I.). Como ele se considerava tímido, trabalhar com computadores o ajudava a "se esconder". Nesta fase, não estava interessado em sair com amigos e preferia permanecer em casa. Com o passar dos anos, perdeu o interesse por seu trabalho. Não via sentido em "trabalhar, pagar contas, viver e morrer". Essa visão negativa parecia resultar do padrão de vida de seu pai: trabalhar duro e se embebedar ao final do dia. $\mathrm{O}$ alcoolismo paterno tinha afetado as relações familiares, rodeadas de tensão e medo. Wilson afirmou que nunca considerou se casar ou ter sua própria casa. Se considerava o protetor de sua mãe.

Ao se questionar sobre a causa de sua timidez, Wilson lembrou de um acidente ocorrido na escola em que sentiu vergonha pelos ferimentos que teve. $\mathrm{O}$ isolamento em casa foi um alívio: estava longe das brigas na escola. $\mathrm{O}$ vínculo tanto com a mãe, quanto com o videogame, se fortaleceu nesta ocasião, tornando-se uma importante fuga das relações interpessoais violentas na escola. Wilson também relatou dificuldades no início da pré-escola, quando apanhou de um garoto e foi estimulado por sua própria família a reagir com agressividade, sempre que ocorresse algo deste tipo.

$\mathrm{Na}$ adolescência, o desafio de se relacionar com as meninas era vivido com sofrimento, pois, embora se sentisse atraído por elas, não conseguia tomar iniciativa de se aproximar. Ele não se achava bonito ou interessante e associava esse sentimento de inferioridade à crença de que sua mãe o considerava feio.

Após cinco meses de consultas, Wilson tinha conseguido superar a vergonha e mostrouse mais disposto a falar sobre assuntos relacionados à sexualidade. Confessou manter o hábito de acessar páginas virtuais de pornografia em busca de um objeto específico de fetiche, e associou esse fetiche a uma cena erótica na infância. Na adolescência, ele descobriu revistas pornográficas com determinada temática fetichista. No entanto, foi no início da idade adulta, com o acesso à internet, que o mundo virtual do fetiche passou a fazer parte de sua rotina diária. As cenas sadomasoquistas lhe ofereciam suporte para um tipo de satisfação sexual inimaginável de ser praticada com mulheres reais.

Wilson esteve em acompanhamento psicológico sob abordagem psicanalítica com periodicidade semanal, durante 9 meses $^{5}$. No início do tratamento, em consulta com um psiquiatra, Wilson tinha sido diagnosticado com depressão grave. Tentou tratamentos com antidepressivos, sem sucesso. No decorrer da análise, o paciente começou a sair de casa para

${ }^{5} \mathrm{O}$ acompanhamento psicológico foi interrompido devido à mudança de trabalho da analista. Desta forma, Wilson foi encaminhado a outro psicólogo.

Doxa: Rev. Bras. Psico. e Educ., Araraquara, v. 21, n. 1, p. 4-16, jan./jun., 2019. 
as consultas e, em alguns meses, retomou encontros esporádicos com amigos. A prática de jogos foi diminuída e conseguiu retomar o hábito de dormir durante a noite e permanecer acordado durante o dia. Foi observada uma melhora em sua capacidade de contar sua própria história com organização temporal. Por outro lado, nesse período, não houve progressos na busca por emprego, tampouco na autonomia pessoal. Wilson avaliou que o acompanhamento psicológico lhe proporcionou ver a vida sob uma melhor perspectiva, já que, desde muito jovem, não via propósito em viver. Em suas palavras: "agora vejo que preciso ter minha própria vida. Antes disso, nem isso eu conseguia ver".

\section{Discussão}

Este é o segundo caso descrito na literatura científica brasileira, que apresenta fortes semelhanças às descrições referentes ao fenômeno hikikomori, conforme apresentadas por Kato et al. (2012); Li e Wong (2015); e Kato, Kanba e Teo (2019). Entretanto, é importante destacar alguns aspectos culturais e psicológicos que ainda não foram devidamente esclarecidos nas pesquisas anteriores sobre o tema. A análise deste caso nos conduziu à hipótese de que, na fase do consumismo virtual da web 2.0, haveria uma exploração das pulsões sexuais voyeuristas e sadomasoquistas, em especial do público masculino por meio de jogos e pornografia, o que poderia favorecer alterações de investimento libidinal dos jovens no que tange às relações intersubjetivas, particularmente entre jovens com vulnerabilidades psíquicas e sociais.

O apego excessivo de Wilson às experiências do ciberespaço parece denotar uma recusa à castração simbólica. Suas afirmações: "no ciberespaço, sou rico, na vida, não sou nada", demonstram um encantamento por fantasias onipotentes. Também evidenciam uma rejeição ao destino paterno: "trabalhar, pagar as contas, viver e morrer", somada à constante embriaguez. Para Wilson, o ciberespaço significava uma alternativa a esse destino insípido, com a promessa de um prazer contínuo, sem culpa e frustrações.

$\mathrm{O}$ caso de Wilson parece ilustrar algumas transformações relacionadas à cultura das sensações midiáticas: seu pai se distraía com álcool, sua mãe vivia suas fantasias por meio de novelas televisivas e Wilson mergulhava em fantasias onipotentes de jogos online e voyeurismo sadomasoquista. Pode se dizer que, os três sujeitos 
experimentavam certo grau de isolamento e apatia, com dificuldade de conferir sentido à própria existência.

Segundo Birman (2009), o fetiche se opõe à tendência pulsional perverso-polimorfa de substituição contínua de objetos. No fetichismo, há uma fixação da pulsão que é cristalizada em um único objeto. Essa imobilidade pulsional pode atingir seu limite em uma mortificação da própria pulsão, cujo desejo permaneceria estagnado (BIRMAN, 2009). Nesse sentido, pode-se supor que uma fixação em uma constante repetição de fantasias onipotentes, por meio dos jogos e das cenas sadomasoquistas das páginas de pornografia, possa ter contribuído, no caso de Wilson, para uma forma de mortificação do desejo, manifestada pela ausência de sentido em viver, bem como por uma busca incessante de preenchimento do vazio interno. Sua satisfação libidinal concentrava-se no voyeurismo sadomasoquista e nos jogos online, sem perspectiva sublimatória.

Poderíamos também concluir que havia uma vulnerabilidade psicológica em Wilson em função de uma fragilidade narcísica, tendo em vista que ele considerava um fardo, ademais, tinha dificuldade em se relacionar afetivamente com o pai, o que pode ter contribuído para a insegurança nas relações fora do círculo familiar. Alguns estudos sobre o hikikomori sugerem traumas de relacionamento na infância, o que poderia resultar na evitação de vínculos afetivos na vida adulta (SAKAMOTO et al., 2005). Ainda sobre as relações familiares, é importante destacar a problemática edipiana e o apego excessivo a sua mãe.

Wilson relatou experiências algumas traumáticas na escola. Sakamoto et al. (2005) identificou uma relação entre hikikomori e bullying escolar. No entanto, no caso de Wilson, a família o estimulava sua agressividade em relação aos colegas, ao invés de buscar resolver os conflitos de maneira pacífica, o que pode ter contribuído para validar as satisfações sadomasoquistas. Com poucas coerções familiares e escolares, no sentido de possibilidades sublimatórias, Wilson se tornou vítima de suas próprias pulsões perversas. Os games violentos em seu quarto consistiam em uma alternativa menos estressante para satisfação dessas pulsões. Para Freud (1930), as coerções familiares e sociais seriam importantes no processo da sublimação das pulsões sexuais e agressivas.

As dificuldades na resolução de conflitos no meio familiar e escolar, o déficit de coerções, bem como os conflitos edípicos contribuíram para uma fixação perverso-polimorfa em satisfações no ciberespaço. Os jogos ofereciam a Wilson uma satisfação alternativa à vida social competitiva, e ao mesmo tempo, permitia uma sociabilidade tecnologicamente controlada, sem riscos. Os fetiches virtuais, por outro lado, poderiam oferecer uma satisfação sexual sem necessidade de se confrontar com a imprevisibilidade dos desejos femininos, nem 
com o risco de experimentar situação de impotência, com a vantagem de não precisar abandonar o casulo maternal.

\section{Considerações finais}

Acredita-se que o caso aqui apresentado pode funcionar como uma lente de aumento sobre aspectos subjacentes à síndrome hikikomori, pois, ao incidir em questões mais profundas da sexualidade, nos oferece pistas para ampliar a compreensão desse fenômeno nos jovens. Porém, não se trata de universalizar as conclusões a partir de um caso único, mas sim de acrescentar novas hipóteses a serem investigadas, considerando a complexidade dos fatores. Ressaltamos a importância de que nas pesquisas sobre hikikomori se considerem as vinculações afetivas na infância e os hábitos virtuais na adolescência, em especial as fantasias perverso-polimorfas. Consideramos provável que as fantasias onipotentes exercitadas no ciberespaço, assim como a excessiva vinculação aos fetiches exibidos nos sites de pornografia, exerçam um importante papel na síndrome hikikomori, embora não sejam suficientes para explicar todos os casos. Uma combinação entre bases narcísicas frágeis, fixação edípica materna, dificuldades no manejo de conflitos escolares, e livre exercício das pulsões perverso-polimorfas fetichistas, voyeurísticas e sadomasoquistas no ciberespaço poderia consistir em fatores de risco; os quais devem ser mais investigados. Também é relevante considerar a transição de uma cultura alicerçada na repressão dos desejos - malestar da cultura -, para o mal-estar dos desejos desmedidos, cuja falta de coerção social no sentido de sublimação das pulsões perversas tem alterado a economia libidinal dos sujeitos, tornando as pessoas mais vulneráveis às fixações fetichistas diversas.

\section{Declaração de conflito de interesse}

Os autores declaram que esta pesquisa foi conduzida sem suporte comercial ou financeiro que possa gerar algum tipo de conflito de interesse.

\section{Agradecimento}

As autoras agradecem a Verlaine Freitas pela discussão do caso. 


\section{REFERÊNCIAS}

ADORNO, T. W.; HORKHEIMER, M. Dialética of enlightenment: philosophical fragments San Francisco: Stanford University Press, 2002.

BIRMAN, J. Pacto perverso e biopolítica. Psicol. Clin. v. 21, n. 2, p. 381-396, 2009.

Disponível em: http://www.scielo.br/scielo.php?pid=S0103-

56652009000200009\&script=sci_abstract\&tlng=pt. Acesso em: 26 ago. 2019.

BOMMERSBACH, T.; MILLARD, H. No longer culture-bound: Hikikomori outside of Japan. Int J. Soc. Psychiatry, v. 65, n. 6, p. 539-540, 2019. Disponível em:

https://journals.sagepub.com/doi/abs/10.1177/0020764019859379. Acesso em: 26 ago. 2019.

BOWKER, J.; BOWKER, M. H.; SANTO, J. B.; OJO, A. A.; ETKIN, R. G.; RAJA, R. Severe social withdrawal: cultural variation in past hikikomori experiences of university students in Nigeria, Singapore, and the United States. J Genet Psychol, p. 1-14, 2019. Disponível em:

https://www.tandfonline.com/doi/abs/10.1080/00221325.2019.1633618? journalCode=vgnt20. Acesso em: 26 ago. 2019.

DOMINGUES-CASTRO, M. S.; TORRES, A. R. Hikikomori: revisão sobre um grave fenômeno de isolamento social. J. bras. Psiquiatr. v. 67, n. 4, p. 264-272, 2018. Disponível em: http://www.scielo.br/scielo.php?script=sci_arttext\&pid=S0047-20852018000400264. Acesso em: 26 ago. 2019.

DUFOUR, D.-R. La cité perverse: liberalisme et pornographie. Paris: Éditions Denoël, 2009.

DUFOUR, D.-R. L’indivu qui vient après le libéralisme. Paris: Éditions Denoël, 2011.

FREUD, S. Three Essays on the Theory of Sexuality. London: Hogarth Press, 1905.

FREUD, S. On narcissism: An introduction. London: Hogarth Press, 1914.

FREUD, S. Fetishism. London: Hogarth Press, 1927.

FREUD, S. Civilization and its Discontents. London: Hogarth Press, 1930.

GONDIM, F. A. A.; ARAGÃO, A. P.; HOLANDA FILHA, J. G.; MESSIAS, E. L. M. Hikikomori in Brazil: 29 years of voluntary social withdrawal. Asian Journal of Psychiatry, v. 30, p. 163-164, 2017. Disponível em: https://www.ncbi.nlm.nih.gov/pubmed/29065363. Acesso em: 26 ago. 2019.

KANEKO, S. Japan's 'socially withdrawn youths' and time constraints in Japanese society: management and conceptualization of time in a support group for hikikomori. Time $\boldsymbol{\&}$ Society. v. 15, p. 233-249, 2006. Disponível em:

https://journals.sagepub.com/doi/10.1177/0961463X06067034. Acesso em: 26 ago. 2019.

KATO, T. A. et al. (2012). Does the 'hikikomori' syndrome of social withdrawal exist outside Japan? A preliminary international investigation. Soc. Psychiatry Psychiatr. 
Epidemiol. v. 47, p. 1061-1075, 2012. Disponível em:

https://www.ncbi.nlm.nih.gov/pubmed/21706238. Acesso em: 26 ago. 2019.

KATO, T. A.; KANBA, S.; TEO, A. R. Hikikomori: Multidimensional understanding, assessment, and future international perspectives. Psychiatri Clin Neurosci, v. 73, n. 8, p. 427-440, 2019. Disponível em: https://www.ncbi.nlm.nih.gov/pubmed/31148350. Acesso em: 26 ago. 2019.

LEBRUN, J-P. La perversion ordinaire: vivre ensemble sans autri. Paris, Denoël, 2007.

LI, T. M. H.; WONG, P. W. C. Youth social withdrawal behavior (hikikomori): A systematic review of qualitative and quantitative studies. Australian and New Zealand Journal of Psychiatry, v. 49, p. 595-609, 2015. Disponível em:

https://www.ncbi.nlm.nih.gov/pubmed/25861794. Acesso em: 26 ago. 2019.

O NAUFRAGO. CAST Direção: Robert Zemeckis. 2000. Título original: Cast Away.

PRIOSTE, C. O adolescente e a internet: laços e embaraços no mundo virtual. São Paulo: edusp, 2016.

SAITO, T. Shakaiteki Hikikomori: Owaranai Shishunki (Social Withdrawal: A Neverending Adolescence). Tokyo: PHP Shinsho, 1998.

SAKAMOTO, N.; MARTIN, R. G.; KUMANO, H.; AL-ADAWI, S. Hikikomori, is it a culture-reactive or culture-bound syndrome? Nidotherapy and a clinical vignette from Oman. International Journal of Psychiatry in Medicine, v. 35, p. 191-198, 2005. Disponível em: https://www.ncbi.nlm.nih.gov/pubmed/16240975. Acesso em: 26 ago. 2019.

SUWA, M.; SUZUKI, K. Psychopathological features of "primary social withdrawal". Seishin Shinkeigaku Zasshi. v. 104, p. 1228-1241, 2002. Disponível em: https://www.ncbi.nlm.nih.gov/pubmed/12649893. Acesso em: 26 ago. 2019.

TEO, A. R.; GAW, A. C. Hikikomori, a Japanese culture-bound syndrome of social withdrawal? A proposal for DSM-5. Journal of Nervous and Mental Disease, v. 198, p. 444-449, 2010. Disponível em: https://www.ncbi.nlm.nih.gov/pubmed/20531124. Acesso em: 26 ago. 2019.

TOIVONEN, T.; NORASAKKUNKIT, V.; UCHIDA, Y. Unable to conform, unwilling to rebel? Youth, culture, and motivation in globalizing Japan. Frontiers in Psychology, v. 2, p. 207, 2011. Disponível em: https://www.ncbi.nlm.nih.gov/pmc/articles/PMC3171786/. Acesso em: 26 ago. 2019.

TÜRCKE, C. Sociedade excitada. Campinas, SP: Editora Unicamp, 2010. 


\section{Como referenciar este artigo}

PRIOSTE, C. D., SIQUEIRA, R. C. Fetichismo virtual na vida de um hikikomori brasileiro: um estudo de caso. Doxa: Rev. Bras. Psico. e Educ., Araraquara, v. 21, n. 1, p. 4-16, jan./jun., 2019. E-ISSN: 2594-8385. DOI: 10.30715/doxa.v21i1.12931

Submetido em: 05/06/2018

Revisões requeridas: 08/09/2018

Aprovado em: 04/12/2018

Publicado em: 01/02/2019 